ORIGINAL ARTICLE

\title{
Hospitalisation for gastroenteritis in Western Australia
}

\author{
M Gracey, A H Lee, K K W Yau
}

Arch Dis Child 2004;89:768-772. doi: 10.1136/adc.2003.037531

See end of article for authors' affiliations

.....................

Correspondence to:

Dr A H Lee, School of

Public Health, Curtin

University of Technology,

GPO Box U 1987, Perth,

WA 6845, Australia:

Andy.Lee@curtin.edu.au

Accepted

11 November 2003

\begin{abstract}
Aims: To document gastroenteritis hospitalisations of the 1995-96 cohort of infants born in Western Australia to mid-2002, and to assess factors associated with their hospitalisations and readmissions. Methods: Retrospective analysis of the State's hospitalisation data, Midwives' Notification of Births data, the Australian Bureau of Statistics mortality data and clinical and demographic information.

Results: Aboriginal infants were hospitalised for gastroenteritis eight times more frequently than their nonAboriginal peers, and were readmitted more frequently and sooner for diarrhoeal illnesses than the other group. They also stayed in hospital for twice as long and many Aboriginal patients were hospitalised on numerous occasions. Hospitalisation rates were higher in remote areas and were significantly associated with co-morbidities such as undernutrition, anaemia, co-existing infections, and intestinal carbohydrate intolerance.

Conclusions: Gastroenteritis is very prevalent in Australian Aboriginal infants and children and is a major cause of their hospitalisation in Western Australia. It is often associated with undernutrition, anaemia, intestinal parasitic infestations, other infections, intestinal carbohydrate intolerance, and, in some instances, with low birth weight. This is often due to unhygienic living conditions and behaviours and presents major challenges to public health, health promotion, and clinical personnel, particularly paediatric services. Childhood diarrhoeal diseases occur commonly in other indigenous groups but have not received the attention that they deserve.
\end{abstract}

G astroenteritis is common in infants and children, particularly where personal, domestic, and community hygiene is unsatisfactory. This is often so in underdeveloped countries ${ }^{1}$ and in other disadvantaged groups, even in otherwise affluent nations. This occurs even in the United Kingdom where a recent commentary mentioned that children from impoverished backgrounds are more commonly affected by infections, particularly those of the respiratory and gastrointestinal tracts. ${ }^{2}$ This also applies among Australia's indigenous population up to 10 years of age whose hospitalisation rates for gastroenteritis are several times those of other Australian children. ${ }^{3}$ Regrettably, many reports about childhood gastroenteritis in vulnerable groups lack data about risk factors associated with their high rates of hospitalisation and re-hospitalisation; these include ethnicity, gender, birth weight, gestational age, locality, nutritional status, and co-existent morbidities such as anaemia, intestinal carbohydrate intolerance, and dehydration.

We are fortunate to be able to examine such comprehensive information in Western Australia (WA) because records of all hospital separations (admissions, transfers, and discharges) are routinely entered into the Hospitals Morbidity Data System of the Health Information Centre of the Department of Health, WA. The Health Information Centre can also effectively link electronic hospitalisation records with maternal and birth characteristics and mortality information. These data are especially valuable because WA covers a vast land mass (2.5 million square $\mathrm{km}$ ), extending from the tropics through subtropical woodlands, very arid deserts, and the more densely populated southwestern agricultural and industrialised areas where most of the non-Aboriginal population lives. The Hospital Morbidity Data System includes whether patients are Aboriginal or not. However, this ethnic definition of patients' origins is not completely reliable. A recent comprehensive study of ethnicity of inpatients in WA showed that $85.5 \%$ of indigenous patients were accurately recorded, State-wide, so the data systematically underestimate the numbers of Aboriginal patients. In the metropolitan health region the proportion identified correctly was $78.3 \%$, while in the remote, tropical Kimberley and Pilbara regions the rate identified correctly was 93.5\%. ${ }^{4}$ Our intention was to document how birth characteristics, nutritional and demographic factors, and comorbidities influenced the readmission patterns for gastroenteritis of a cohort of Aboriginal infants born in WA during 1995-96 compared with a contemporary non-Aboriginal cohort.

\section{SUBJECTS AND METHODS}

The sources of data for this retrospective cohort study are the WA Hospital Morbidity Data System, the WA Midwives' Notification of Births, and the Australian Bureau of Statistics mortality database. Linked hospitalisation, birth, and death records of each individual from these sources are extracted by the Health Information Centre of the Department of Health, WA. Record linkage is used to retrieve the medical history of each patient from the diagnostic information recorded on hospital separation summaries. A unique patient number is attached by record linkage to all records for each individual to facilitate retrieval of information. Linkage of hospitalisation records permits determination of readmissions from multiple records of each individual, and provides birth, morbidity, and mortality information for patients during their episodes of care.

All infants born in WA in 1995 and 1996 who had an index gastroenteritis admission during their first year of life were included. This cohort was followed through until 31 May 2002 to document their subsequent hospitalisation patterns. There were no deaths in this group over that time which were attributed to gastroenteritis or its complications. The total number of patients studied was 1001 after removing the eight deaths from other causes; this means that each infant in the cohort had approximately the same follow up period (exposure). All hospital records with a principal diagnosis of diarrhoea (ICD-9 and ICD-10 clinical codes 001-009) and diagnosis related groups (DRG) 350 and 351 were retrieved 
Table 1 Causes of diarrhoea included with ICD-9 and ICD-10* clinical codes 001-009 and diagnosis related groups (DRG) 350 and 351

\begin{tabular}{ll}
\hline $\begin{array}{l}\text { Cholera } \\
\text { Typhoid fever }\end{array}$ & $\begin{array}{l}\text { Amoebic infestations } \\
\text { Giardiasis } \\
\text { Paratyphoid fever }\end{array}$ \\
$\begin{array}{l}\text { Other salmonella infections } \\
\text { Other specified protozoal intestinal } \\
\text { diseases } \\
\text { Intestinal viral infections } \\
\text { Shigellosis }\end{array}$ & $\begin{array}{l}\text { Intestinal infections due to other } \\
\text { organisms }\end{array}$ \\
$\begin{array}{l}\text { Other specified bacterial } \\
\text { Bnfections }\end{array}$ & Ill defined intestinal infections \\
(unspecified) & Diarrhoea of presumed infectious origin \\
Bacterial food poisoning & *There was a change from ICD-9-CM codes to ICD-10-AM in July 1999.
\end{tabular}

for the birth cohort. There was a change from ICD-9-CM to ICD-10-AM in July 1999. These diagnostic categories cover the majority of causes of episodes of infectious diarrhoea (see table 1).

Co-morbidities during hospitalisation were also documented using the ICD-9 and ICD-10 codes. Intestinal carbohydrate intolerance was detected and recorded if the patient had persistent fluid diarrhoea when on a diet containing sugars, most often lactose, and with evidence of acid stools, a positive Clinitest examination of the fluid part of a fresh stool specimen, and a clinical response to elimination of lactose or other relevant carbohydrate from the diet. ${ }^{5}$ Nutritional status was assessed after initial rehydration with reference to World Health Organisation (WHO) international reference values.

The following outcome measures are considered:

- Readmission rate: (number of readmissions since index admission)/(exposure in years), where exposure is defined as the period from the separation date of index admission to the census date 31 May 2002

- Readmission proportion: (number of readmitted patients)/ (total number of patients)

- Time to first readmission: time from the separation date of index admission to the first readmission (in weeks)

- Length of stay: length of hospital stay per patient (in days).

All data were coded and analysed using SPSS version 11. To assess the differences in readmission rate, time to first readmission and length of stay among nominal risk factors, the Mann-Whitney test (for two groups) and Kruskal-Wallis test (for three or more groups) were used. To assess the association between categorical factors and the readmission occurrence, the Pearson $\chi^{2}$ test was used instead.

\section{RESULTS}

\section{General description of the findings}

There were 50 641 live births in WA during 1995 and 1996; of these, 2870 (5.7\%) were Aboriginal. In total there were 1001 patients in the original birth cohort, of whom 317 (32\%) were Aboriginal. Table 2 presents the demographic and descriptive statistics of the original birth cohort. Eleven per cent of the Aboriginal birth cohort were admitted to hospital for gastroenteritis compared with only $1.4 \%$ of the nonAboriginal cohort. Aboriginal patients comprised 38.3\% of the 1295 gastroenteritis admissions. Aboriginal patients made up $32 \%$ of all index admissions and $55.7 \%$ of patients who were admitted more than once. The admission and readmission patterns were examined according to each of WA's seven health service regions (see fig 1).

Approximately $90 \%$ of all admissions were classified as "emergencies" and $35.7 \%$ of Aboriginal patients were

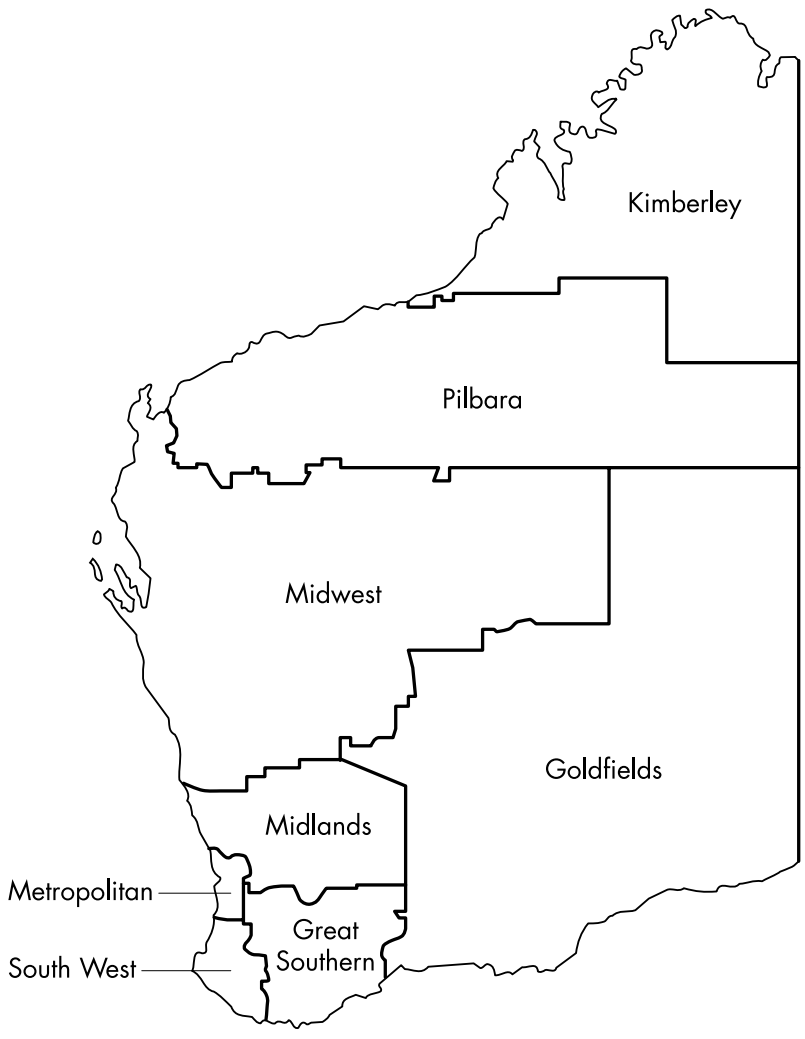

Figure 1 Map of Western Australia showing its health service regions. The State covers the western third of the Australian Continent and has a land area of approximately 2.5 million square $\mathrm{km}$. At its widest dimensions it measures about $2200 \mathrm{~km}$ from north to south and $1500 \mathrm{~km}$ from east to west.

seriously dehydrated on admission compared with only $23.7 \%$ of non-Aboriginal patients. Undernutrition $(<3 \mathrm{rd}$ centile of WHO reference values) was present in $12.5 \%$ of Aboriginal patients but in only $3.6 \%$ of non-Aboriginal patients; $7.5 \%$ of the Aborigines but only $0.9 \%$ of the other patients had iron deficiency anaemia. Gastrointestinal carbohydrate intolerance occurred in $9.5 \%$ of Aborigines but among only $5 \%$ of non-Aborigines.

The relative proportion of Aboriginal:non-Aboriginal patients who were readmitted was 2.7 and the relative rates of Aboriginal:non-Aboriginal admissions was 3.4. The mean time to readmission of Aboriginal patients was 33 (SD 44.1) weeks and for the other patients was 62.4 (SD 65.6) weeks. The mean length of hospital stay (LOS) for Aborigines was 4.8 (SD 5.7) days and for non-Aboriginal patients was 2.2 (SD 3.4) days; mean LOS for readmission episodes was 5 (SD 6.8) days for Aborigines and 2.4 (SD 2) days for nonAborigines.

\section{Readmission rates}

These were significantly different $(\mathrm{p}<0.001)$ among the health regions; the highest readmission rate $(0.122)$ was in the very remote Kimberley region in the far north. Rates were also high in the Midwest (rate $=0.077)$, Pilbara (rate $=0.068)$, and Goldfields (rate $=0.047)$ regions. By contrast, the rate in the metropolitan area of Perth and surrounds was 0.036. For Aboriginal and non-Aboriginal patients combined, readmission rates were much higher in country rather than metropolitan areas $(p<0.001)$; rates $=0.074$ and 0.036 , respectively. Aboriginality was highly correlated with readmission rates $(\mathrm{p}<0.001)$ and male infants were readmitted more frequently than females 
Table 2 Patient demographic and descriptive statistics

\begin{tabular}{|c|c|c|c|}
\hline & Overall & Aboriginal & Non-Aboriginal \\
\hline Number of gastroenteritis admissions & 1295 & 496 & 799 \\
\hline Number of patients & 1001 & 317 & 684 \\
\hline Number of patients hospitalised once & 782 & 195 & 587 \\
\hline Number of patients with readmissions & 219 & 122 & 97 \\
\hline Average number of admissions & 1.29 & 1.57 & 1.17 \\
\hline Maximum number of admissions & 6 & 6 & 5 \\
\hline Readmission rate & 0.055 & 0.106 & 0.031 \\
\hline Readmission proportion & 0.219 & 0.385 & 0.142 \\
\hline Mean time to first readmission in weeks (SD) & $46.1(56.5)$ & $33.0(44.1)$ & $62.4(65.6)$ \\
\hline Mean LOS in days (SD) & $3.04(4.40)$ & $4.78(5.68)$ & $2.24(3.37)$ \\
\hline Mean readmission LOS in days (SD) & $3.82(5.41)$ & $4.98(6.82)$ & $2.37(1.98)$ \\
\hline \multicolumn{4}{|l|}{ Proportion of patients (\%) } \\
\hline Male & 55.6 & 58.4 & 54.4 \\
\hline Aboriginal & 31.7 & 100.0 & 0.0 \\
\hline Non-metropolitan residence & 49.1 & 78.2 & 35.5 \\
\hline Low birth weight $(<2.5 \mathrm{~kg})$ & 12.5 & 16.4 & 10.7 \\
\hline Premature birth (gestational age $<37$ weeks) & 22.4 & 24.8 & 21.4 \\
\hline \multicolumn{4}{|l|}{ Proportion of admissions (\%) } \\
\hline Emergency admission & 91.3 & 94.0 & 89.5 \\
\hline Dehydration & 28.3 & 35.7 & 23.7 \\
\hline Gastrointestinal sugar intolerance & 6.7 & 9.5 & 5.0 \\
\hline Undernutrition & 7.0 & 12.5 & 3.6 \\
\hline Iron deficiency anaemia & 3.4 & 7.5 & 0.9 \\
\hline Failure to thrive and anaemia & 9.7 & 18.3 & 4.3 \\
\hline Infection (genitourinary/scabies/otitis media) & 13.4 & 24.0 & 6.9 \\
\hline
\end{tabular}

$(\mathrm{p}<0.05)$. Low birth weight $(<2500 \mathrm{~g})$ and early gestational age $(<37$ weeks) were not significantly correlated with readmission rates. Undernutrition (body mass $<3$ rd centile of WHO international reference values) was highly correlated with readmission rates $(p<0.001)$, and readmission rates were twice as high among patients who had iron deficiency anaemia during their index episode of hospitalisation $(p<0.05)$. The presence of genitourinary tract infections, scabies, or otitis media, and the occurrence of dehydration during hospitalisation did not influence subsequent readmission to hospital.

\section{Length of hospital stay}

The mean length of hospital stay (LOS) per patient, including the index episode of hospitalisation, for the whole State, was 3 days; LOS differed significantly between the health regions $(\mathrm{p}<0.001)$. The longest mean LOS of 5 days occurred in the Kimberley. Aboriginality was highly correlated with mean LOS $(\mathrm{p}<0.001)$; on average, Aboriginal patients stayed in hospital for 4.8 days while other patients stayed only 2.2 days. Low birth weight (LBW) babies stayed significantly longer (3.8 versus 2.8 days) than babies of normal birth weight $(p<0.001)$, but gender and gestational age had no significant associations with LOS. The presence of other infections, such as of the skin, middle ears, or genitourinary tracts (all are prevalent in Aboriginal children) were highly significant $(p<0.001)$ in affecting LOS; those children with such infections stayed, on average, for 5.6 days while patients without them were hospitalised for only 2.7 days. Undernutrition $(p<0.001)$, the occurrence of intestinal carbohydrate intolerance $(\mathrm{p}<0.001)$, and the presence of clinically significant dehydration $(\mathrm{p}<0.001)$ all had statistically important impacts on prolonging the LOS. Iron deficiency anaemia also prolonged the period of hospitalisation ( 8.9 versus 2.9 days; $\mathrm{p}<0.001$ ).

Patterns of LOS during readmission were significantly different in the various health regions $(\mathrm{p}<0.005)$; patients in the Kimberley stayed in hospital twice as long as those in the metropolitan area (6 versus 3 days) and Aboriginal patients stayed in hospital, on average, for 5 days versus 2.4 days for non-Aboriginal patients, State-wide. Undernutrition (7.4 versus 3.3 days; $p<0.001$ ), the occurrence of intestinal carbohydrate intolerance at first admission (8.5 versus 3.4 days; $\mathrm{p}<0.005)$, and the presence of co-existing infections during the index admission episode (5.7 versus 3.4 days; $\mathrm{p}<0.01$ ) were significantly associated with subsequent length of hospital stay during readmission for gastroenteritis. Birth weight, gestational age at birth, and the occurrence of clinically significant dehydration during the index episode of gastroenteritis did not significantly affect subsequent length of hospital stay for gastroenteritis readmissions. Patients with iron deficiency anaemia were hospitalised for twice as long ( 10.3 versus 3.5 days), but this was not statistically significant, probably because of the small numbers of patients involved.

\section{Readmission patterns}

State-wide, 219 patients (294 episodes) of the original 1001 patients were readmitted with a primary diagnosis of gastroenteritis during the study period; some patients were readmitted on multiple occasions. In the Kimberley $41 \%$ of patients were readmitted while in the Midwest and Pilbara regions the proportions were $32 \%$ and $28 \%$, respectively. Twenty eight per cent of all patients in country areas were readmitted, while only $16 \%$ of those living in the metropolitan area were readmitted. All of these differences in readmission patterns by locality were highly significant $(\mathrm{p}<0.001)$. Aboriginality was correlated with readmission patterns (39\% versus 14\%; p < 0.001 ) and readmissions were significantly more frequent among males than females $(24 \%$ versus $19 \% ; p<0.05)$. Undernutrition $(37 \%$ versus $21 \%$; $\mathrm{p}<0.001)$ and the presence of iron deficiency anaemia $(38 \%$ versus $21 \%$; $p<0.05$ ) were significantly associated with subsequent readmission for gastroenteritis. Birth weight, gestational age, and the occurrence of clinically significant dehydration, intestinal carbohydrate intolerance, and the presence of co-existing infections during the index episode did not have any significant bearing on subsequent hospitalisation for gastroenteritis. 


\section{Time to first readmission}

Overall, the mean time to first readmission was 46 weeks; this was significantly shorter for Aboriginal than for other patients (33 versus 62.4 weeks; $p<0.001$ ). Mean time to readmission was shorter for patients with intestinal carbohydrate intolerance ( 24 versus 48 weeks; $p<0.005$ ) and for patients with serious dehydration during their index admission (35 versus 50 weeks; $\mathrm{p}<0.05$ ).

\section{DISCUSSION}

Aboriginal infants and children were hospitalised for gastroenteritis approximately eight times more frequently in Western Australia than were their non-Aboriginal peers from the late 1990s to mid-2002. This resembles the findings of a recent study ${ }^{3}$ and, again, shows the heavy infectious diseases burden experienced by this seriously disadvantaged group in this nation. This inequity in Australian child health has been recognised for decades, ${ }^{6-8}$ although there has been some decline in absolute, compared with relative (Aboriginal: nonAboriginal) hospitalisation rates for gastroenteritis over the past decade and a marked decline in deaths due to diarrhoeal disease in infants and children in WA between 1970 and $2000 .^{3}$

Perhaps the most important feature of this study is the identification of patients by Aboriginality which enabled us to compare and correlate different aspects of hospitalisation and readmission between the two groups. For example, almost $36 \%$ of the Aborigines compared with $24 \%$ of nonAborigines were severely dehydrated on admission; this may be due to more severe illness among Aboriginal patients complicated by undernutrition, late presentation for clinical care, and the long distances many Aboriginal people live from hospitals. Undernutrition was much more common among Aboriginals (12.5\%) than among other patients $(3.6 \%)$ as were intestinal carbohydrate intolerance ( 9.5 versus $5 \%$ ) and iron deficiency anaemia ( 7.5 versus $<1 \%)$.

The relative proportion of Aboriginal patients who were readmitted was more than double that of the other group and their relative rate of readmission was more than three times that of the non-Aboriginal cohort. This is probably due to their higher rates of complications, longer distances from clinical care, and the difficulties that are often experienced in adequately supervising post-hospitalisation follow up of Aboriginal patients, particularly in remote areas, where patients may live hundreds of kilometres from the nearest hospital. Readmissions were commoner in regional and remote areas than in the city, among Aborigines than other patients, and were more frequent in patients who, during their index hospital admission, were poorly nourished, or had iron deficiency anaemia, intestinal carbohydrate intolerance, or co-existent infections. The average time to readmission for gastroenteritis among Aboriginal patients (33 weeks) was almost half of that for the other patients (62 weeks), and the mean LOS among Aborigines (4.8 days) was more than double that of other patients (2.2 days); a similar pattern occurred during readmissions. Time elapsed before readmission was significantly affected by Aboriginality, intestinal carbohydrate intolerance, and severe dehydration during the index episode of hospitalisation.

As mentioned, gastroenteritis has been recognised as a serious disease among young Australian Aborigines for decades. $^{3-9}$ This is largely due to the unsatisfactory, unhygienic, and overcrowded living conditions in which many of these people and their families live; that exposes them to high levels of microbiological environmental contamination and increases the risk of faecally transmitted diseases, including gastroenteritis, from human and animal sources and from other intermediate disease carrying vectors including flies and larger vermin as well as contaminated food and water. ${ }^{10}$ Worldwide, childhood diarrhoeal diseases continue to be a major health problem and cause more than two million deaths annually; ${ }^{11}$ this is a particular burden in underdeveloped countries but also occurs in industrialised nations. In the United States, for example, childhood diarrhoea causes more than 200000 episodes of hospitalisation and 300 deaths in under-5s annually and has an economic cost of US\$2 billion. ${ }^{12}$

Gastroenteritis as a serious health issue in indigenous populations has received less attention internationally than is warranted. This may be partly due to inadequate appreciation of the particular socioeconomic, political, health, and related factors that influence the lives of indigenous people and negatively affect their health. Gastroenteritis was recognised as a serious problem in the White Mountain Apaches in Arizona in the early 1970s. ${ }^{13}$ Other reports have shown that Native American children, including those from Alaskan Native populations, are especially prone to gastrointestinal infections, ${ }^{14-16}$ similar to our experience and that of others working among Australian Aborigines. The valuable information from North America was facilitated by reliable data from the long established Indian Health Service and tribal hospital discharge records as well as the National Discharge Survey data. Such information is extremely useful because it enables the assessment of inequities in hospitalisations between different ethnic groups as is certainly the case with Aboriginal versus other Australians of all ages for various illnesses. ${ }^{17}$ Other indigenous populations have high rates of childhood gastroenteritis, for example in Mayan Indians in Mexico, in Ethiopia, and among Ciskeian villagers in South Africa, ${ }^{18-20}$ and almost certainly in many other indigenous populations. Comparative information of this type allows the documentation of differences in hospitalisation usage patterns and provides opportunities to develop strategies as to why these exist and to develop ways to correct inequities in health in vulnerable groups. But it must be appreciated that hospitalisation rates depend on many factors and do not necessarily directly indicate levels of morbidity in different groups. These factors can include, for example, local or regional hospitalisation policies, the availability of skilled clinical staff in local clinics or hospitals, the likelihood of reliable post-hospitalisation compliance with medications or other follow up care, and the distances that patients live from and have access to hospital care.

Despite these caveats, we have shown that gastroenteritis is much more serious in young Aborigines than among their non-Aboriginal peers in WA. Several factors and comorbidities correlate with these higher rates of hospitalisation and with more frequent and rapid readmission for gastroenteritis. These findings have significant public health implications as well as messages for health promotion and disease prevention and the provision of better access to high standard clinical care for youngsters who are particularly vulnerable to gastroenteritis, especially in indigenous populations.

\section{ACKNOWLEDGEMENTS}

We are indebted to Diana Rosman, William Pyper, and Peter Somerford of the Department of Health, Western Australia for providing data, coding information, and the map of Western Australia, respectively. We are most grateful to the Office of Aboriginal Health of the Department of Health, Western Australia, for supporting this project.

\section{Authors' affiliations}

M Gracey, A H Lee, School of Public Health, Curtin University of Technology, Perth, Western Australia

K K W Yau, Department of Management Sciences, City University of Hong Kong, Hong Kong 


\section{REFERENCES}

1 Gracey M. Diarrhea and malnutrition: a challenge for pediatricians. J Pediatr Gastrenterol Nutr 1996;22:6-16.

2 Reading R. Poverty and the health of children and adolescents. Arch Dis Child 1997;76:463-7.

3 Gracey M, Cullinane J. Gastroenteritis and environmental health among Aboriginal infants and children in Western Australia. J Paediatr Child Health 2003;39:427-31

4 Young MJ. Assessing the quality of identification of Aboriginal and Torres Strait Islander People in Western Australian Hospital Data, 2000. Health Information Occasional Paper 13, ISSN 1329 7252. Perth: Health Department of Western Australia, 2001.

5 Gracey M, Anderson CM. Disorders of carbohydrate digestion and absorption. In: Gracey M, Burke V, eds. Pediatric gastroenterology and hepatology, 3rd edn. Boston: Blackwell Scientific Publications, 1993:380-402.

6 Moodie PM. Mortality and morbidity in Australian Aboriginal children. Med J Aust 1969;1:180-5.

7 Jose DG, Welch JS. Growth retardation, anaemia and infection, with malabsorption and infestation of the bowel. The syndrome of protein-calorie malnutrition in Australian Aboriginal children. Med J Aust 1970;1:349-56.

8 Gracey M. Enteric disease in young Australian Aborigines. Aust N Z J Med 1973;3:576-9.

9 Gracey M. Diarrhoea in Australian Aborigines. Aust J Public Health 1992; 16:216-25

10 Gracey M, Williams P, Houston S. Environmental health conditions in remote and rural Aboriginal communities in Western Australia. Aust N Z J Public Health 1997;21:511-18.
11 UNICEF. The state of the world's children. Oxford, UK: Oxford University Press, 1998.

12 American Academy of Pediatrics. Practice parameter: the management of acute gastroenteritis in young children. American Academy of Pediatrics, Provisional Committee on Quality Improvement, Subcommittee on Acute Gastroenteritis. Pediatrics 1996;97:424-35.

13 Hirschhorn N, Cash, Woodward W, et al. Oral therapy of Apache children with acute infectious diarrhoea. Lancet 1972;1:15-18.

14 Santosham M, Moulton LH, Perez M, et al. Efficacy and safety of high-dose rhesus-human reassortant rotavirus vaccine in Native American populations. J Pediatr 1997;131:632-8

15 Moulton LH, Staat MA, Santosham M, et al. The protective effectiveness of natural rotavirus infection in an American Indian population. $J$ Infect Dis 1998; 178:1562-6

16 Holman R, Curns AT, Cheek JE, et al. Infectious disease hospitalizations among American Indian and Alaska Native infants. Abstract e176. Pediatrics 2003;111:422.

17 Torzillo P, Kerr C. Contemporary issues in Aboriginal public health. In: Reid J, Trompf $\mathrm{P}$, eds. The health of Aboriginal Australia. Sydney: Harcourt, Brace, Jovanovich, 1991:326-80.

18 Granich R, Cantwell MF, Long K, et al. Patterns of health seeking behavior during episodes of childhood diarrhea: a study of Tzotzil-speaking Mayans in the highlands of Chiapas, Mexico. Soc Sci Med 1999;48:489-95.

19 Tenssay ZW, Mengistu A. Bacterial isolates from indigenous weaning foods in rural Ethiopian setting, Jimma Zone, south west Ethiopia. Ethiopian Med J 1997:35:93-102.

20 Segar J. Hard lives and evil winds: illness aetiology and the search for healing amongst Ciskeian villagers. Soc Sci Med 1997;44:1585-600

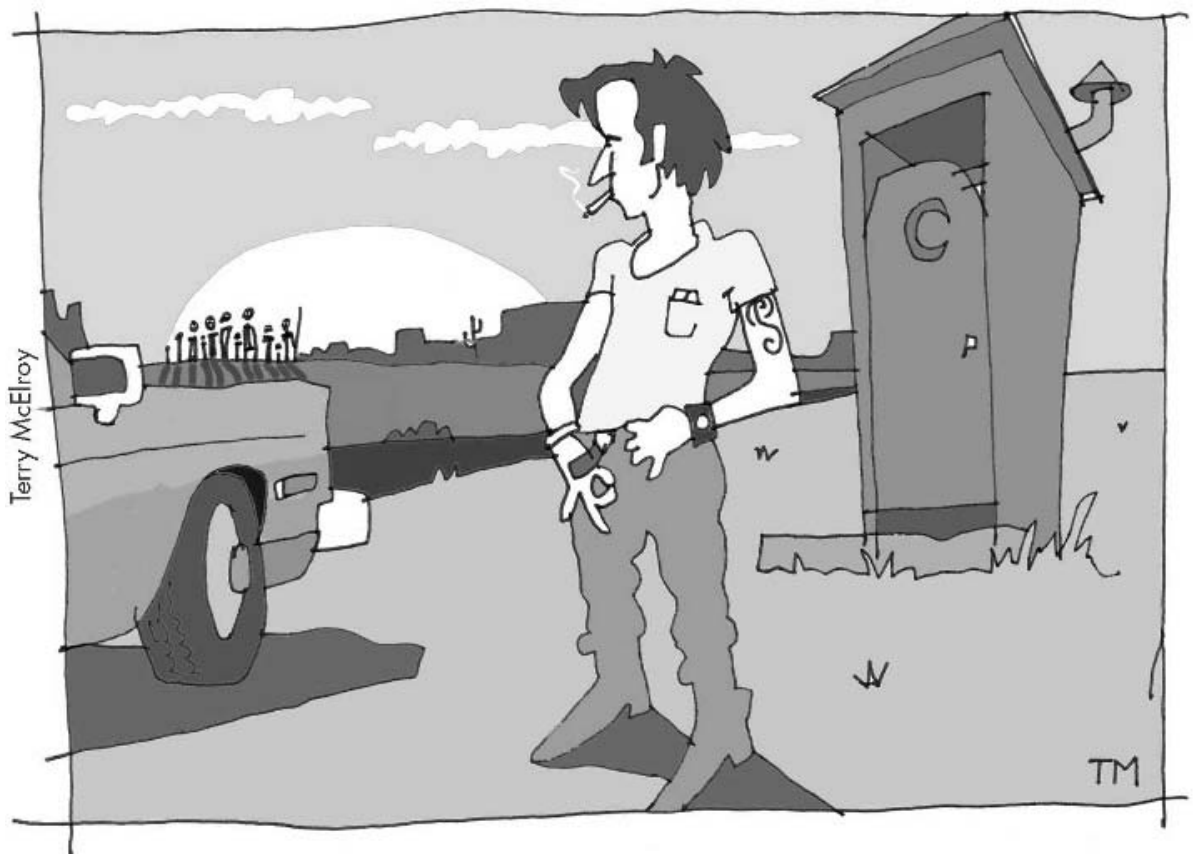

